\title{
Promotion of Adhesion Between Resin and Silica-coated Titanium by Silane Monomers and Formic Acid Catalyst
}

\author{
Jukka P. Matinlinna • Lippo V. Lassila • Jon E. Dahl
}

Received: 29 March 2010 / Accepted: 3 May 2010 / Published online: 22 May 2010

(C) The Author(s) 2010. This article is published with open access at Springerlink.com

\begin{abstract}
Three bifunctional silane coupling agent monomers and their blends with a cross-linking silane were investigated as six experimental adhesion-promoters in vitro. Three organosilane monomers, 3-methacryloxypropyltrimethoxysilane, styrylethyltrimethoxysilane, and 3-(N-allylamino)propyltrimethoxysilane, were prepared as $1 \%(\mathrm{v} / \mathrm{v})$ primers and also blended with a cross-linking silane, 1,2-bis-(triethoxysilyl)ethane at $0.5 \%(\mathrm{v} / \mathrm{v})$, in a 95:5 solution of ethanol in deionized water. The silanes were allowed to hydrolyze using formic acid as catalyst and were applied to a substrate of silica-coated Ti. The control was a prehydrolyzed silane coupling agent that is commonly used in dentistry. An experimental unfilled bis-phenol-A-diglycidyldimethacrylate/methylmethacrylate resin was bonded by photo-polymerization as stubs to the prepared Ti. Half of the specimens were artificially aged by wet thermocycling and half were stored in dry conditions in a desiccator. Storage conditions and the type of primer silane or blend significantly affected shear bond strength values. 3Methacryloxypropyltrimethoxysilane, alone or blended, pro-
\end{abstract}

J. P. Matinlinna $(\bowtie)$

Dental Materials Science, Faculty of Dentistry,

The University of Hong Kong, The Prince Philip Dental Hospital,

34 Hospital Road,

Hong Kong SAR, People's Republic of China

e-mail: jpmat@hku.hk

\section{V. Lassila}

Department of Biomaterials Science, Institute of Dentistry,

The University of Turku,

Lemminkäisenkatu 2,

FI-20520 Turku, Finland

J. E. Dahl

Nordic Institute of Dental Materials (NIOM),

P.O. Box 79,

NO-1305 Haslum, Norway duced significantly higher shear bond strength values than the control, both after dry storage and after thermo-cycling.

Keywords Formic acid · Silica-coating · Silica · Silane coupling agent $\cdot$ Silane monomer $\cdot$ Titanium

\section{Introduction}

Silicon dioxide (silica) and silane coupling agents have several applications in clinical dentistry. Metallic restorations, such as substructures, crowns and bridges are silica-coated and silanized before veneering or cementation [1, 2]. Furthermore, bifunctional silicon esters are applied as adhesion promoters between dissimilar materials, such as inorganic materials to organic polymers. These silane coupling agents are synthetic compounds with characteristic direct covalent carbon-silicon bonds. They have hydrolyzable alkoxy groups attached to their Si atom, with ethoxy groups being kinetically much slower to hydrolyze than methoxy groups [3]. 3-Methacryloxypropyltrimethoxysilane, also known as 3-methacryloyloxypropyltrimethoxysilane, is indicated as a pre-hydrolyzed ready-to-use primer (adhesion promoter) in dental technology and prosthodontics for silicacoated materials, see Fig. $1[4,5]$.

Initially, hydrophobic silane monomers are inactive and thus need to be activated by hydrolysis, typically by water/ ethanol. The reaction requires a catalyst, normally acetic acid $[3,4]$. The corresponding alcohol is released during the reaction, while the silane monomers become labile intermediary hydrophilic silanols that oligomerize and deposit onto the silica-coated substrate. In so doing, silane coupling agents can effectively promote wetting of the substrate surface. The non-cured siloxane film that forms on the inorganic substrate can then react with an organic phase [6]. However, the 
<smiles>C=C(C)C(=O)OCCC[Si](OC)(OC)OC</smiles>

a)<smiles>C=CCNCCC[Si](OC)(OC)OC</smiles>

c)<smiles>C=Cc1ccc(CC[Si](OC)(OC)OC)cc1</smiles>

b)

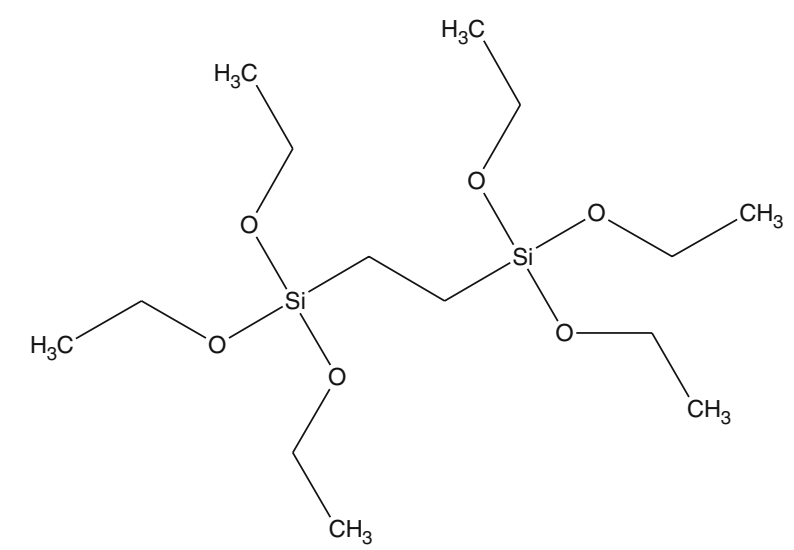

d)

Fig. 1 Silane coupling agent monomers: a 3-Methacryloxypropyltrimethoxysilane, b Styrylethyltrimethoxysilane, c 3-(N-allylamino) propyltrimethoxysilane, $\mathbf{d}$ 1,2-bis-(triethoxysilyl)ethane (non-functional cross-linking silane)

hydrolytic stability of silane-aided bonding, and hence the service time of the restoration in the hostile oral environment (i.e. with humidity, sudden $\mathrm{pH}$ and temperature changes, bruxism and biting forces), is a topic of concern [3-5].

Blends of bifunctional silane and cross-linking silane monomers have been reported to achieve considerable hydrolytic stability and to promote adhesion in applications of the tyre and steel manufacturing industries [7-9]. The most usual cross-linking silane is bis-1,2-(triethoxysilyl)ethane, which has six hydrolyzable ethoxy groups (Fig. 1). The siloxane film that forms during curing is extensively branched and traps water and gas molecules (Fig. 2). An interpenetrating polymer network is understood to form between the silanized substrate and organic coatings or resin-composites [3, 7-11].

Titanium is an excellent biomaterial that exhibits unique osseointegration in implant dentistry and is used in bridge or crown substructures $[12,13]$. The authors recently reported results from the application of a novel silane system on silica-coated titanium, i.e. a bifunctional organofunctional silane combined with a cross-linking silane [14, 15]. Various organofunctional groups in silane esters may have different reactivities with monomers of a resin-composite or resin only. The spatial match of the silane molecule can also vary. In addition, the authors have investigated experimental silane primers as adhesion promoters for polished $\mathrm{Ti}$ [16] and silica-coated $\mathrm{Ti}$ [17]. In vitro studies of novel silane systems have suggested that their significantly enhanced adhesion could be useful in dental materials applications.

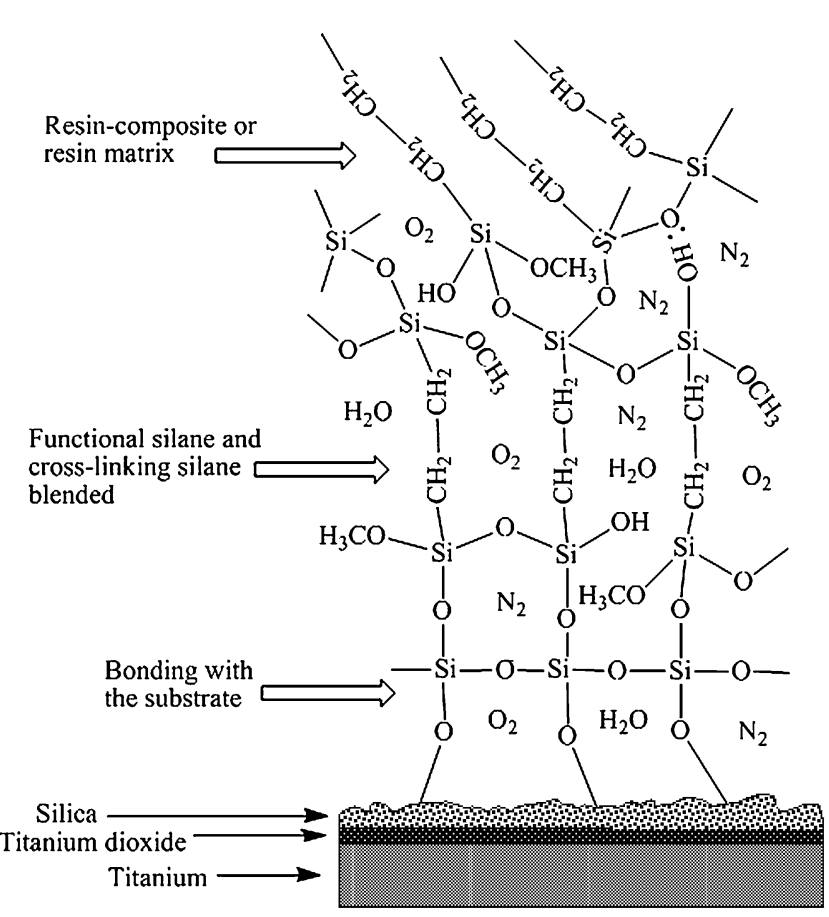

Fig. 2 Silane-aided bonding and the supposed siloxane layers on silica-coated Ti substrate when an organofunctional silane is blended with a cross-linking silane 
This in vitro study continues our investigations on silane monomer systems and the use of a non-filled experimental resin for dental purposes [14, 15]. The medium for all experimental primers was again the optimized 95:5 ratio of ethanol in deionized water [16, 17], but now acidified with formic acid, $\mathrm{HCOOH}$. As a cross-linking silane, bis-1,2(triethoxysilyl)ethane was selected on the basis of previous results [18-21]. This study used functional silanes of 3methacryloxypropyltrimethoxysilane, 3-(N-allylamino) propyltrimethoxysilane and styrylethyltrimethoxysilane because they all have a vinylic $>\mathrm{C}=\mathrm{C}<$ group in a different chemical context, which contributes to their differential reactivity (Fig. 1a-c). The control silane in this study, a pre-activated (pre-hydrolyzed) silane coupling agent product contained ca. 2 vol\% 3-methacryloxypropyltrimethoxysilane, had a $\mathrm{pH}$ of ca. 5, and $>90 \%$ ethanol, catalyzed (and inhibited) with unknown chemicals, and without formic acid [21]. The control was used as such, without any modification to make an objective comparison between it and the experimental silane primers. The first hypothesis was that formic acid-catalyzed activation of silane monomers and their blends with bis-1,2-(triethoxysilyl)ethane, produces stronger bonding between the resin and silica-coated $\mathrm{Ti}$ than the control silane. The second hypothesis was that artificial wet aging by thermo-cycling to simulate oral conditions weakens bonding to different extents depending on the primer.

\section{Experimental}

The materials used in the experimental primers are listed in Table 1 . The other study materials were the same as those used in a previous study; the experimental resin was also prepared as described previously, see also below [14].
Commercially pure grade 2 Ti (Permascand, Ljungaverk, Sweden; $>99 \%$ ) of $1-\mathrm{mm}$ thickness was cut into rectangular coupons of $20 \mathrm{~mm} \times 40 \mathrm{~mm}(n=28)$. The upper horizontal half of the surface was grit-blasted with silica-coated alumina sand (Rocatec $^{\mathrm{TM}}$ Plus, 3M ESPE, Seefeld, Germany) at $300 \mathrm{kPa}$ for $10 \mathrm{~s}$ from a perpendicular distance of $10 \mathrm{~mm}$. The silica-coated $\mathrm{Ti}$ coupons were cleaned in ethanol in an ultrasonic bath, air-dried at room temperature for $15 \mathrm{~min}$ and randomly divided into two study groups, reflecting two storage conditions.

Six experimental primers were prepared in a $95 \%(\mathrm{v} / \mathrm{v})$ solution of ethanol in deionized water, which was acidified with $1 \mathrm{M}$ formic acid to produce a final $\mathrm{pH}$ of 4.5 . First, a $0.5 \%$ (v/v) solution of bis-1,2-(triethoxysilyl)ethane was prepared in the ethanol/water mix and allowed to activate by hydrolysis for $23 \mathrm{~h}$ at room temperature. Then, each organofunctional silane was added to reach $1.0 \%$, and the blends were allowed to stabilize for an additional $1 \mathrm{~h}$ at room temperature before use. Organofunctional silanes without the bis-1,2-(triethoxysilyl)ethane were also prepared and activated at room temperature. A pre-hydrolyzed commercially available silane coupling agent was used as the control [4, 5, 21].

The six primers and control were each applied with a fine brush as a single coat onto silica-coated $\mathrm{Ti}$ coupons and were allowed to dry for $3 \mathrm{~min}$. Silica-coating was performed in a standardized way by the same researcher. An experimental unfilled bis-phenol-A-diglycidyldimethacrylate/methylmethacrylate and methylmethacrylate, bisGMA/MMA, resin [14] was transferred into polythene molds to form resin stubs with a diameter of $3.6 \mathrm{~mm}$ and height of $4 \mathrm{~mm}$. Five resin stubs were evenly placed on the upper horizontal borders of the silica-coated Ti coupons. The stubs were then photo-polymerized with an Optilux 501 lamp (SDS Kerr, Danbury, USA) at $490 \mathrm{~mW} \mathrm{~cm}^{-2}$ for
Fig. 3 Shear bond strength (MPa) after dry storage ("Dry") and after thermo-cycling ("TC6000"). Key: STYRX= Styrylethyltrimethoxysilane, $\mathrm{ALAP}=3-(\mathrm{N}$-allylamino)propyltrimethoxysilane, MPS =3-methacryloxypropyltrimethoxysilane, $\mathrm{BTSE}=1,2-$-bis-(triethoxysilyl) ethane

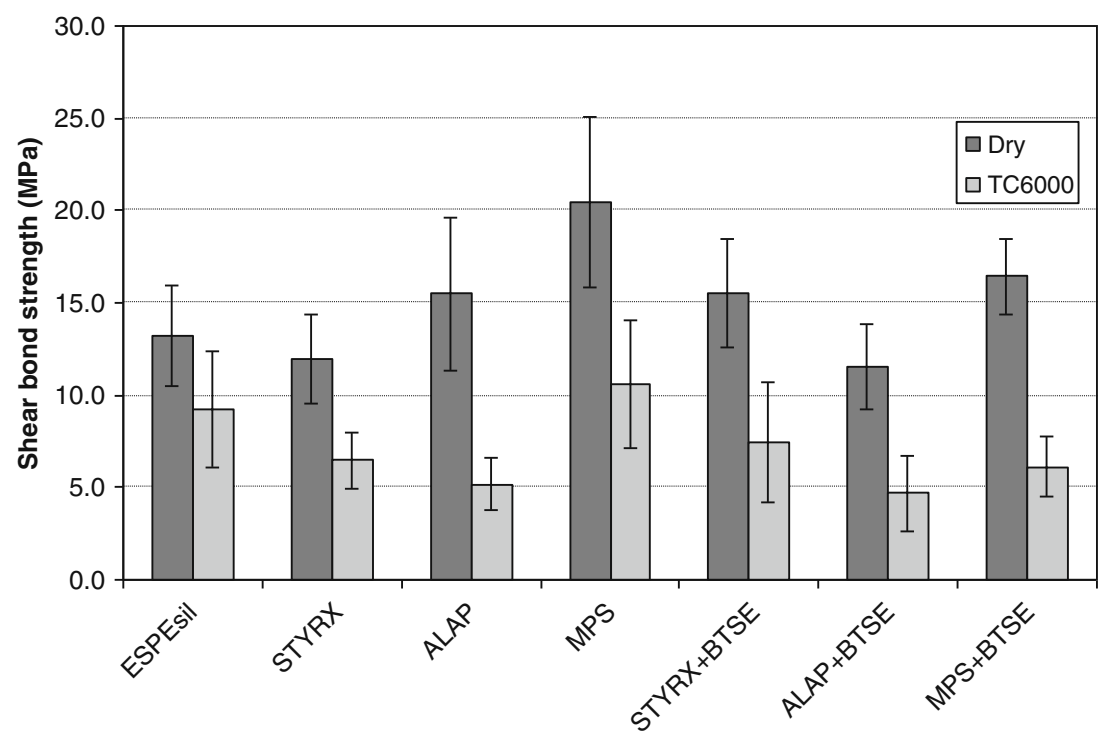


Table 1 Materials used in this study

\begin{tabular}{lllll}
\hline Material, brand & Abbreviation & Manufacturer & Purity [\%] & Batch number \\
\hline ESPE Sil silane coupling agent & Control silane & 3M ESPE, Seefeld, Germany & N/A & 256464 \\
Ethanolum Anhydricum & Ethanol & Arcus, Oslo, Norway & 99.5 & 030305 \\
Formic acid & HCOOH & Merck, Darmstadt, Germany & $98-100$ & K34234564 503 \\
1,2-bis-(triethoxysilyl)ethane & BTSE & Gelest, Tullytown, PA, USA & 100 & $4 \mathrm{R}-4325$ \\
Styrylethyltrimethoxysilane & STYRX & Gelest, Morrisville, PA, USA & 92 & $7 \mathrm{D}-10467-5$ \\
3-(N-allylamino)propyltrimethoxysilane & ALAP & ABCR, Karlsruhe, Germany & 95 & 5 D-6650-Y \\
3-Methacryloxypropyltrimethoxysilane & MPS & Dow Corning, Midland, MI, USA & $>95$ & 0002015730 \\
\hline
\end{tabular}

40 s. A final polymerization step was immediately performed in a Visio ${ }^{\circledR}$ BETA Vario photo-curing vacuum unit (ESPE, Seefeld, Germany) for $15 \mathrm{~min}$, to ensure adequate polymerization, as is routinely performed in dental laboratories. The specimen mold was removed while the cured resin stubs were pressed perpendicularly against the Ti coupon with a steel instrument. One half of the prepared Ti coupons were kept in a desiccator for $24 \mathrm{~h}$. The other half were subjected to wet thermo-cycling for 6,000 cycles between $5.0^{\circ} \mathrm{C}\left( \pm 0.5^{\circ} \mathrm{C}\right)$ and $55.0^{\circ} \mathrm{C}\left( \pm 0.5^{\circ} \mathrm{C}\right)$, with a standard dwelling time of $30 \mathrm{~s}$ and a transfer time of $2 \mathrm{~s}$, in a custom-made system (modified Heto CBN 18-30 water baths; Allerø, Denmark). Immediately before testing, all specimens were immersed in a water bath at $37^{\circ} \mathrm{C}$ to prevent drying [14-22].

Shear bond strength testing was carried out at a constant cross-head speed of $1.0 \mathrm{~mm} \mathrm{~min}^{-1}$ until fracture occurred at the resin-Ti interface, using a universal testing machine (LRX®; Lloyd Instruments, Fareham, UK). The shear bond strength (in $\mathrm{MPa}$ ) was calculated by dividing the highest force (in $\mathrm{N}$ ) needed, by the contact area (in $\mathrm{mm}^{2}$ ) of the resin stub [23]. Thermo-cycled specimens were examined by optical microscopy (at a magnification of 50-100) to assess the failure type in terms of resin remaining on the $\mathrm{Ti}$ surface. When $33 \%$ of the resin stub remained on the $\mathrm{Ti}$ surface, the failure type was assigned "adhesive", when $>33 \%$ but $<66 \%$ remained, it was "mixed failure" and when $>66 \%$ remained, it was "cohesive" $[14,15]$.

Differences in shear bond strength results between test groups were assessed with analysis of variance (ANOVA), using the Statistical Package for the Social Sciences (SPSS, Chicago, IL, USA). Multiple comparisons were performed with Tukey's test, Scheffe's test, and Levene's test of equality of error variances. Statistical significance was set at $p=$ 0.001. Post hoc tests were not performed for storage mode, because there were fewer than three storage conditions.

\section{Results and Discussion}

The results (Fig. 3) showed that all silane primers tested except styrylethyltrimethoxysilane alone and 3-(N-allyla- mino)propyltrimethoxysilane + bis-1,2-(triethoxysilyl)ethane resulted in higher shear bond strength values than the control under dry storage conditions (Table 2). Although thermo-cycling has a somewhat controversial role in shear bond strength testing [24], it was used to simulate oral conditions and led to lower bond strength values than all corresponding dry-storage samples. The highest shear bond strength was obtained with treatment by 3methacryloxypropyltrimethoxysilane alone, both for specimens that underwent dry storage (19.4 MPa [5.6 MPa]) and for those that underwent thermo-cycling (10.6 MPa [SD, 3.6 MPa]). These values were $15.2 \%$ and $48.1 \%$ higher, respectively, than those achieved by the control silane. The lowest shear bond strength for drystorage specimens was 11.5 $\mathrm{MPa}(2.8 \mathrm{MPa})$, which was obtained with styrylethyltrimethoxysilane, indicating $12.2 \%$ weaker bonding than the control. The next lowest for dry-storage specimens, at $9.9 \%$ weaker bonding, was demonstrated after using a blend of 3-(N-allylamino) propyltrimethoxysilane with bis-1,2-(triethoxysilyl)ethane. This blend also exhibited the lowest shear bond strength value among thermo-cycled specimens, at $4.7 \mathrm{MPa}$ (2.1 $\mathrm{MPa}$ ), which represented a $48.9 \%$ reduction in bonding strength compared with the control. No spontaneous debonding was observed after thermo-cycling (Table 2). All failures for samples treated with styrylethyltrimethoxysilane and 3-(N-allylamino)propyltrimethoxysilane, and their blends, were adhesive failures. Most cases of failure for the control were cohesive and some were mixed, but none showed adhesive failure. In contrast, 3-methacryloxypropyltrimethoxysilane and 3methacryloxypropyltrimethoxysilane + bis-1,2-(triethoxysilyl)ethane led to all three failure types observed.

Of the thermo-cycled specimens, only those silanized with 3-methacryloxypropyltrimethoxysilane alone exhibited greater shear bond strength than the control. ANOVA (Table 3) showed significant differences in shear bond strength values between storage type $(p<0.001)$ and silane blend $(p<0.001)$. Levene's test showed that error variance of the dependent variable was not equal across specimen groups: $\mathrm{F}=2.435$, $\mathrm{dfl}=13, \mathrm{df} 2=123, p=0.006$. The substantial reduction in 
Table 2 Shear bond strength (mean, standard deviation) for specimens under two storage conditions, change in bonding strength and the failure mode after thermo-cycling ( $n=10$ per group). Key: SBS=Shear bond strength, $\mathrm{SD}=$ standard deviation, MPS=3-methacryloxypropyltrimethoxysilane, BTSE $=1,2$-bis-(triethoxysilyl)ethane, STYRX=styrylethyltrimethoxysilane, and $\mathrm{ALAP}=3$-(N-allylamino)propyltrimethoxysilane

\begin{tabular}{|c|c|c|c|c|c|c|c|c|}
\hline \multirow[t]{2}{*}{ Silane } & \multicolumn{2}{|c|}{ Dry storage: } & \multicolumn{6}{|c|}{ Thermo-cycled: } \\
\hline & $\begin{array}{l}\text { SBS (SD) } \\
{[\mathrm{MPa}]}\end{array}$ & $\begin{array}{l}\text { Change } \\
{[\%]}\end{array}$ & $\begin{array}{l}\text { SBS (SD) } \\
{[\mathrm{MPa}]}\end{array}$ & $\begin{array}{l}\text { Change } \\
{[\%]}\end{array}$ & $\begin{array}{l}\text { Spontaneous } \\
\text { debonding }[\%]\end{array}$ & $\begin{array}{l}\text { Cohesive } \\
\text { failure }[\%]\end{array}$ & $\begin{array}{l}\text { Mixed } \\
\text { failure [\%] }\end{array}$ & $\begin{array}{l}\text { Adhesive } \\
\text { failure [\%] }\end{array}$ \\
\hline Control ESPE Sil & $13.1(2.9)$ & Reference & $9.2(3.3)$ & Reference & 0 & 80 & 20 & 0 \\
\hline MPS & $19.4(5.6)$ & 48.1 & $10.6(3.6)$ & 15.2 & 0 & 50 & 40 & 10 \\
\hline MPS+BTSE & $16.7(2.2)$ & 27.5 & $6.1(1.7)$ & -33.7 & 0 & 20 & 40 & 40 \\
\hline STYRX & $11.5(2.8)$ & -12.2 & $6.5(1.6)$ & -29.3 & 0 & 0 & 0 & 100 \\
\hline STYRX+BTSE & $15.0(3.4)$ & 14.5 & $7.4(3.4)$ & -19.6 & 0 & 0 & 0 & 100 \\
\hline ALAP & $14.7(4.8)$ & 12.2 & $5.2(1.5)$ & -43.5 & 0 & 0 & 0 & 100 \\
\hline $\mathrm{ALAP}+\mathrm{BTSE}$ & $11.8(2.5)$ & -9.9 & $4.7(2.1)$ & -48.9 & 0 & 0 & 0 & 100 \\
\hline
\end{tabular}

bond strength values due to thermo-cycling for all specimen groups can be partially explained by the more than 6-fold difference between thermal expansion coefficients of the experimental methacrylate resin (approximately the value for acrylic denture resin, about $76 /{ }^{\circ} \mathrm{C} \times 10^{6}$ ) and $\mathrm{Ti}$, (about $12{ }^{\circ}$ $\left.\mathrm{C} \times 10^{6}\right)[25]$.

The shear bond strength results obtained in this study are lower than those reported in our previous studies of resin bonding to silica-coated $\mathrm{Ti}[14-16,18]$, as well as those in studies in which a silane blend produced significantly stronger bonding that functional silanes alone [18, 20, 21]. The three bifunctional silanes in this study were used without further purification by re-distillation, which may have contributed to reduced performance. However, all three had methoxy groups connected to a Si atom, which suggests that there should not be any remarkable differences in their kinetic hydrolysis, although the latter was not investigated. In addition, these silane monomers were visually assessed as being good for use, as they were not hazy or cloudy before the activation step, which would have indicated autopolymerization due to ambient humidity. It is thus likely that the differences in the linkers between $\mathrm{C}=\mathrm{C}$ and $\mathrm{Si}$ (Fig. 1a-c) account for the different primer performances.

The most widely applied silane coupling agent, 3methacryloxypropyltrimethoxysilane, is used to prepare unsaturated polyester-fiberglass composites [3, 10], and as a copolymer with styrene in sol-gel synthesis and in dental composites [3-6]. Silanes with aromatic styryl functionality are typically used as coupling agents for unsaturated polyesters and for phenolic and epoxy fiberglass laminates, as well as in the production of printed circuit boards. On the other hand, 3-(N-allylamino)propyltrimethoxysilane has applications as a coupling agent for polyesters and for acrylic coatings of glass containers [10, 26]. The bisfunctional silane bis-1,2-(triethoxysilyl)ethane has two Si atoms with hydrolysable ethoxy groups; it is thus nonfunctional. bis-1,2-(Triethoxysilyl)ethane is used in relatively low concentrations as an additive in silane coupling agent blends in order to enhance hydrolytic stability in applications such as paint primers and coatings for corrosion protection [7-9]. It has also produced significantly enhanced results in silane-aided promotion of adhesion in in vitro simulations of dental applications [18-21].

A hydrolysis time of $23 \mathrm{~h}$ was selected for the crosslinking silane and $1 \mathrm{~h}$ was selected for functional silanes because functional trialkoxy silanes hydrolyze sufficiently within $1 \mathrm{~h}$ [16-21]. Formic acid was used as the catalyst, and it has some similarities with acetic acid, which has been used in past studies. Both appear as dimers in aqueous solution, owing to hydrogen bonding. Formic acid protonates somewhat more easily and has a $\mathrm{pK}_{\mathrm{a}}$ of 3.75, corresponding to a $\mathrm{K}_{\mathrm{a}}$ value of $1.8 \times 10^{-4}$, whereas acetic
Table 3 Statistical analysis (ANOVA): between-subject effects and results of two-way ANOVA for the shear bond strength of the experimental silane primers. Dependent variable: shear bond strength

${ }^{\mathrm{a}} \mathrm{R}$ Squared $=0.679$ (Adjusted Squared $=0.645$ )

\begin{tabular}{lcrrrr}
\hline Source & Sum of Squares & df & Mean Square & \multicolumn{1}{c}{ F } & $p$ Value \\
\hline Corrected Model & $2667.231^{\mathrm{a}}$ & 13 & 205.172 & 19.985 & 0.000 \\
Storage & 1928.819 & 1 & 1928.819 & 187.878 & 0.000 \\
Silane Blend & 580.019 & 1 & 96.670 & 9.416 & 0.000 \\
Storage * Silane Blend & 164.684 & 6 & 27.447 & 2.674 & 0.018 \\
Error & 1262.760 & 123 & 10.266 & & \\
Total & 20274.266 & 137 & & & \\
Corrected Total & 3929.992 & 136 & & & \\
\hline
\end{tabular}


acid has a $\mathrm{pK}_{\mathrm{a}}$ of 4.75 , corresponding to a $\mathrm{K}_{\mathrm{a}}$ value of $1.8 \times$ $10^{-5}$ [27]. Acetic acid has a wider selection of applications in industry as a solvent and material for chemical synthesis. Formic acid is a strong reducing agent and is used as an acidulant in the dyeing of synthetic and natural fibers, leather tanning, and in chemical synthesis [28]. The high reduction potential and acidity of formic acid may explain the unexpected weaker resin-Ti bonding when formic acid was used as a $\mathrm{pH}$ regulator and hydrolysis catalyst in the experimental primers. For example, formic acid and styrylethyltrimethoxysilane as a coupling agent for silicacoated Ti were not as effective (reactive) in this study as when acetic acid was used with styrylethyltrimethoxysilane as a coupling agent for silica-coated zirconia ceramic [29]. Formic acid may thus have somehow inhibited the primer monomers either during or after hydrolysis. Chemical interaction between the silane oligomers and formic acid dimers or formate ions may have also affected or modified the wetting of the silica-coated Ti surface. Another variable is the solvent system. Isopropanol, acidified with acetic acid to $\mathrm{pH} 4$ has been reported as an alternative solvent to 3-methacryloxypropyltrimethoxysilane [30]. Some aprotic solvents for silanes, such as pentane, xylene, $n$-heptane or cyclohexane have been assessed previously by others, but they used a catalyst e.g. n-propylamine [31]. Effects of varying the solvent system for experimental silane primers need to be investigated in the future.

The experimental primers with 3-methacryloxypropyltrimethoxysilane and styrylethyltrimethoxysilane and their blends, but not 3-(N-allylamino)propyltrimethoxysilane and its blend, produced shear bond strength values after thermo-cycling that exceeded the critical threshold of $5 \mathrm{MPa}$, set by the ISO Standard 10477 Amendment for dental materials [23]. This finding suggests potential development of 3-methacryloxypropyltrimethoxysilane and styrylethyltrimethoxysilane primer systems for clinical use. The control silane, which contained ca. 2 vol\% of 3-methacryloxypropyltrimethoxysilane was a fresh, unopened bottle to maximize its effect. It had a shelf-life of three years, reported by the manufacturer. This kind of silane is prehydrolyzed, i.e. it contains oligomers and its futher autopolymerization is controlled using an inhibitor, most probably an amino compound. A completetly autopolymerized silane product without adhesion promotion properties could be visually recognized: the solution would have turned to hazy and cloudy in the bottle [21]. Now the control silane, which contained 3-methacryloxypropyltrimethoxysilane and was a fresh, unopened bottle of a type of silane coupling agent in regular use in clinical dentistry [4, 22], also resulted in bonding values that exceeded the critical threshold, which contrasts with findings of our previous study [14, 15]. Because the experimental 3methacryloxypropyltrimethoxysilane primer was freshly prepared and applied already after a hydrolysis within $1 \mathrm{~h}$, it was more reactive with the silica-coated surface than the control with its oligomeric silane species fragments. Its effective shelf-life without deterioration can be up to one year as suggested [32].

Finally, although we did not analyze the chemical composition of the silica-coated $\mathrm{Ti}$ surface or investigate the roughness of the surface, these factors certainly have their effects on the adhesion process. Nevertheless, it is mandatory that silica-coating pretreatment for bonding of resins to metals in dentistry should be followed by silane application. It was proposed more than 20 years ago that the bonding mechanisms of silane coupling agents were well understood [33]. In contemporary silane research, the statement no longer holds true.

\section{Conclusions}

We have investigated the effects of three silanes alone, in ethanol/water acidified with formic acid, and in combination with a cross-linking silane on resin-Ti adhesion. All silane primers tested except styrylethyltrimethoxysilane alone and 3-(N-allylamino)propyltrimethoxysilane + bis-1,2-(triethoxysilyl)ethane increased shear bond strength values under dry-storage conditions. Thermo-cycling significantly lowered all shear bond strength values for all silanes and blends tested. Only 3-methacryloxypropyltrimethoxysilane, alone or blended, produced significantly higher shear bond strength values than the control, both after dry storage and after wet specimen storage conditions, i.e. thermo-cycling.

Acknowledgements The Nordic Institute of Dental Materials (NIOM, Oslo, Norway) and The Finnish Dental Society Apollonia are acknowledged for generous research and conference travel grants. Dr. Barry Arkles (Gelest Inc., Tullytown, PA, USA) is thanked for generously donating the silane monomers and Dr. Trevor Lane (The University of Hong Kong) for his kind assistance in proofreading this manuscript.

Open Access This article is distributed under the terms of the Creative Commons Attribution Noncommercial License which permits any noncommercial use, distribution, and reproduction in any medium, provided the original author(s) and source are credited.

\section{References}

1. Kern M, Thompson VP (1993) Dent Mater 9:155

2. Heikkinen TT, Lassila LVJ, Matinlinna JP, Vallittu PK (2009) Silicon 1:199

3. Plueddemann EP (1970) J Adhes 2:184

4. Matinlinna J, Lassila LVJ, Özcan M, Yli-Urpo A, Vallittu PK (2004) Int J Prosthodont 17:155

5. Matinlinna JP, Vallittu PK (2007) J Contemp Dent Pract 8:1

6. Nihei T, Kurata S, Kondo Y, Umemoto K, Yoshino N, Teranaka T (2002) J Dent Res 81:482 
7. Sundararajan PG, van Ooij WJ (2000) Surf Eng 16:315

8. van Ooij WJ, Zhu DQ, Prasad G, Jayaseelan S, Fu Y, Teredesai N (2000) Surf Eng 16:386

9. Seth A, van Ooij WJ, Puomi P, Yin Z, Ashirgade A, Bafna S, Shivane C (2007) Prog Org Coat 58:136

10. Rosen MR (1978) J Coat Technol 50:70

11. Klempner D, Sperling LH, Utracki LA (1994) Interpenetrating polymer network. Advances in chemical sciences. Am Chem Soc, Washington vol 239

12. Steinemann SG (1998) Periodontol 2000 17:7

13. Buser D, Belser UC, Lang NP (1998) Periodontol 2000 17:106

14. Matinlinna JP, Lassila LVJ, Vallittu PK (2010) Silicon 1(4):249

15. Matinlinna JP, Lassila LVJ (2010) Silicon. doi:10.1007/s12633010-9039-6

16. Matinlinna J, Özcan M, Lassila LVJ, Vallittu PK (2004) Dent Mater 20:804

17. Matinlinna JP, Lassila LVJ, Kangasniemi I, Vallittu PK (2005) J Dent Res 84:360

18. Matinlinna JP, Özcan M, Lassila LVJ, Kalk W, Vallittu PK (2008) Acta Odontol Scand 66:250

19. Matinlinna JP, Lassila LVJ, Vallittu PK (2007) Acta Odontol Scand 65:44
20. Matinlinna JP, Lassila LVJ, Vallittu PK (2006) J Dent 34:436

21. Matinlinna JP, Lassila LVJ, Vallittu PK (2006) J Dent 34:740

22. Matinlinna JP, Lassila LVJ, Vallittu PK (2006) J Dent 34:721

23. International Organization for Standardization (ISO) (1996) Dentistry-Polymer-Based Crown and Bridge Materials, Amendment 1996; ISO 10477

24. Söderholm K-J (2009) J Adhes Sci Technol 23:973

25. O'Brien (2002) Dental materials and their selection, 3rd edn. Quintessence, Carol Stream, pp 313-314

26. Arkles B (1977) Chemtech 7:542

27. Atkins P (2001) The elements of physical chemistry. Oxford University Press. 3rd edition, p 169

28. O'Neil MJ (2006) The Merck Index, 14th Edition, Merck Research Laboratories, Whitehouse Station, NJ, USA

29. Matinlinna JP, Lassila LVJ, Vallittu PK (2008) PEF IADR Abstracts, London, UK, \#0279, p 118

30. Puska M, Lassila L, Seppälä J, Vallittu P, Matinlinna J (2009) J Adhes Sci Technol 23:991

31. Chen TM, Brauer GM (1982) J Dent Res 61:1439

32. Hooshmand T, van Noort R, Keshvad A (2004) Dent Mater 20:635

33. Roulet JF (1987) Degradation of dental polymers. Karger, Basel, p 18 